\title{
An Ingenious Application-Specific Quality Assessment Methods for Compressed Wireless Capsule Endoscopy Images
}

\author{
Kinde Anlay Fante, Fetulhak Abdurahman and Mulugeta Tegegn Gemeda \\ Faculty of Electrical and Computer Engineering, Jimma Institute of Technology, Jimma University, Ethiopia
}

\begin{abstract}
Image quality assessment methods are used in different image processing applications. Among them, image compression and image super-resolution can be mentioned in wireless capsule endoscopy (WCE) applications. The existing image compression algorithms for WCE employ the generalpurpose image quality assessment (IQA) methods to evaluate the quality of the compressed image. Due to the specific nature of the images captured by WCE, the general-purpose IQA methods are not optimal and give less correlated results to that of subjective IQA (visual perception). This paper presents improved image quality assessment techniques for wireless capsule endoscopy applications. The proposed objective IQA methods are obtained by modifying the existing full-reference image quality assessment techniques. The modification is done by excluding the noninformative regions, in endoscopic images, in the computation of IQA metrics. The experimental results demonstrate that the proposed IQA method gives an improved peak signal-tonoise ratio (PSNR) and structural similarity index (SSIM). The proposed image quality assessment methods are more reliable for compressed endoscopic capsule images.
\end{abstract}

Index Terms-Image quality assessment, wireless capsule endoscope, compressed images, image compression.

\section{INTRODUCTION}

Wireless capsule endoscopy (WCE) is a tablet size camera with a radio frequency transmitter which can be easily ingested by patients to diagnose gastrointestinal abnormalities [1]. It enables the non-invasive imaging of the gastrointestinal tract with a high-resolution camera operating in the visible electromagnetic spectrum. The capsule captures the image of the inner wall of the human digestive system and transmits it to a data recorder located outside the patient's body. The transmission of high-resolution images via a wireless link consumes high power and requires a high transmission bandwidth [2]. In order to reduce the power consumption and transmission bandwidth, image compression algorithms are included inside the capsule [3]. Image processing software on workstation is used to decompress and process the received images in order to improve the visual quality. Among the image processing methods, super-resolution algorithms [4] are proposed to improve the spatial resolution of the images, image post-processing and error-correcting codes are proposed to suppress the distortion due to transmission channel noise [5], and computer-aided diagnosis systems [6] are used to improve the detection of abnormalities during the evaluation phase.

Generally, high quality images are required for accurate diagnosis of abnormalities. Ideally, lossless image compression methods are needed to retain all information in medical images. However, the lossless image compression algorithms achieve a low compression rate. It cannot significantly reduce the power consumption and the bandwidth requirement of the WCE transmission system. Hence, lossy image compression algorithms are preferred to achieve a high compression rate. The amount of information loss due to the compression process should not affect the diagnosis outcome [7]. On the other hand, image compression algorithms that have high information loss achieve a high compression rate. A good compromise between the image quality and compression rate needs to be maintained in this particular application.

In order to design an image compression algorithm that can achieve a high compression ratio without affecting the diagnosis accuracy, we need reliable image quality assessment methods. Such methods can help us to optimize the parameters of the image compression algorithms, such as quantizer values and sub-sampling factors [2]. The image quality assessment methods can be categorized into two groups: the subjective and objective quality assessment methods [8]. The subjective quality assessment method is more accurate, time-consuming, and expensive. Hence, the objective image quality assessment methods are widely used in practice. The objective image quality assessment methods are widely used to quantify the quality of medical images in compression algorithms [2], [3]. Among the popular methods, the peak signal-to-noise ratio (PSNR) [9], structure similarity index (SSIM) [10], and feature similarity index (FSIM) [11] were employed to evaluate the distortion introduced due to the quantization operation of the endoscopic image compression algorithms. In literature, a minimum peak signal-to-noise ratio (PSNR) value of $30 \mathrm{~dB}$ has been considered to be sufficient for accurate diagnosis of pathology in endoscopic images [12]. However, in [7], [13], a PSNR value of $35 \mathrm{~dB}$ was considered as a threshold value for an accurate diagnosis of abnormalities using medical images. A reliable objective IQA method for WCE application will help to preserve the information needed for accurate diagnosis of abnormalities and optimize the parameters of image compression algorithms. Some of these IQA methods, for instance, PSNR and SSIM, give equal importance to all parts of the image in their evaluation of the image quality assessment metrics. However, the quality of all the parts of the image may not be equally important in some applications. For example, in an endoscopic capsule image, the four corners of the image are non-informative regions. The pixels in those regions are all zero. These regions have no important 
information, and the quality of the pixels in these regions is unaffected by the image compression algorithms. This makes the existing objective image quality assessment techniques less reliable for WCE application, and their correlation with the subjective assessment techniques is low. In this work, we present an improved objective image quality assessment methods that exclude the impact of the non-informative regions. The proposed ad-hoc algorithm improves the performance of general-purpose algorithms by incorporating knowledge about the nature of the images. The incorporated knowledge helps these algorithms to completely remove the impact of noninformative regions in the IQA metrics calculation.

The existing objective IQA methods can be classified into three categories based on the availability of reference images [14]. These are full reference, reduced reference, and nonreference IQA methods. The focus of this paper is on full reference IQA methods for compressed endoscopic capsule images. The full reference IQA methods can be further divided into two broad categories. The fist category uses statistical error metrics such as mean square error (MSE), PSNR, and visual signal-to-noise ratio (VSNR), etc. And the second category takes into account the knowledge of the human visual system (HVS), which includes FSIM, SSIM, Visual Information Fidelity (VIF), etc. These IQA metrics are general-purpose methods and may not be suitable for some applications. An example is an image taken using a capsule camera, as shown in Fig. 1. Due to the circular shape of the lens of the capsule camera and the rectangular shape of the image sensor arrays, the four corners of the WCE image are occluded from the illumination source. The corner regions of this image have no important information. and, all of its pixel values are zero. It also creates a sharp transition boundary between the corner regions and the circular visual region of the image. We can put forward the following two observations from the nature of endoscopic capsule images. First, the quality of the four corner regions of endoscopic images is unaffected by lossy image compression algorithms since all pixel values are zeros. This affects the statistical error based image quality metrics such as MSE and PSNR. Second, the sharp transition boundary between the corner regions (non-informative regions) and circular visual region of the endoscopic capsule image is highly affected by lossy image compression algorithms that involve sub-sampling and quantization such as DCT-based [15] and DWT-based [16] algorithms. Similarly, the quantization and subsampling operation in DPCM-based [17], [18], and error due to inadequate side information in distributed video coding algorithms [19]. However, this region has no significant information relevant to the diagnosis of abnormalities in the gastrointestinal tract. Most of the IQA methods that use the knowledge about the human visual system, such as FSIM, SSIM, VIF, etc., estimate the information content of image regions based on the variance of the image blocks. An image block that lies in the boundary between the non-informative regions and the circular visual region has a very high variance. The presence of this high variance region significantly affects the reliability of the IQA metrics for this particular domain of images.

Generally, we can use three types of knowledge in the design of image quality assessment methods. These are pieces of knowledge about the human visual system (HVS), statistics of the image, and the distortion type [14].

The previous works on this topic assessed the suitablity of the general-purpose image quality assessment methods for WCE application. Both subjective and objective image quality assessment methods were presented [20]-[22]. However, the objective image quality assessment methods for compressed WCE images [20] have not taken into consideration the peculiar nature of the WCE images. By using the knowledge about the WCE camera, we developed more reliable image quality assessment methods. In this work, we use the knowledge about the statistics of the image acquisition system to improve the performance of the general-purpose objective IQA methods for wireless capsule endoscopy applications. The contribution of this work is that it excludes non-informative regions in the computation of objective image quality assessment methods for WCE application. To the best of our knowledge, this the first work which takes into consideration the peculiar nature of WCE images in the image quality assessment method for WCE application. The remaining sections of the paper are organized as follows. Section II discusses the analysis of the proposed IQA method. In Section III, the result and discussions are given. Finally, the conclusion is given in Section IV.

\section{Analysis of the Proposed Image Quality Assessment Methods}

The proposed IQA algorithm improves the performance of the general-purpose IQA methods by excluding the following two regions in the computation of the IQA metrics. These are: (1) the non-informative (corner) regions, and (2) the boundary between the corner regions and the circular visual region. This section will investigate the overall impact of excluding these two regions in the computation of general-purpose IQA metrics for WCE application. The block diagram of a full-

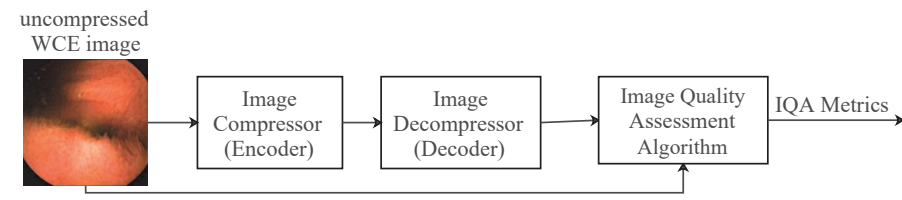

Fig. 1. Block diagram of a full-reference objective image quality assessment method for compressed WCE Images.

reference image quality assessment method is depicted in Fig. 1. The image is compressed to reduce its size for storage or transmission. The lossy image compression process introduces distortion to the image. In both DPCM-based [2] and DCT-based [23] image compression algorithms, the chroma subsampling and quantization processes introduce distortion. The compressed image is then decompressed (decoded) for visualization. The amount of distortion in the decompressed is quantified using image quality assessment method. The image quality assessment method takes both the decoded image and the reference image to compute the objective image quality assessment metrics. 


\section{A. The Effect of Corner Regions}

As shown in Fig. 2, the visual region of the capsule camera is the circular region. For the image of size $W \times W$, the area of the visual region is $\frac{\pi W^{2}}{4}$, and the area of the whole image is $W^{2}$. The total area of the non-informative regions, $A_{n i}$, is given by:

$$
A_{n i}=W^{2}\left(1-\frac{\pi}{4}\right)
$$

For $W=256, A_{n i}=14064$ pixels. This is about 21.46 $\%$ of the total pixels of the image, which constitutes nearly $220,8 \times 8$ patches. The total number of pixels in the noninformative region is more than one-fifth of the total numbers of pixels of the image, and the impact on the image quality assessment metrics must be considered. The exclusion of the pixels in the non-informative regions in the computation of image quality assessment metrics improves the reliability of the methods. Several image quality assessment methods

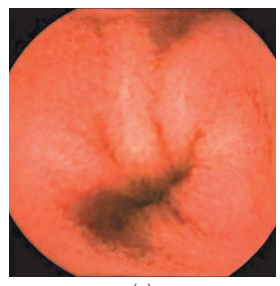

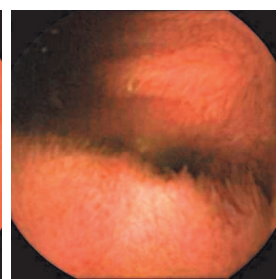

(b)

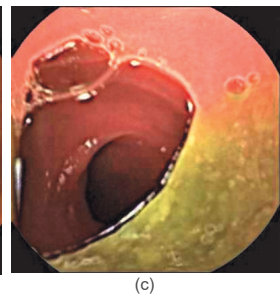

(c)
Fig. 2. Wireless capsule endoscopy images [24].

were used to evaluate the performance of wireless capsule endoscopy image compression algorithms. Among them, mean squared error (MSE) and peak signal-to-noise ratio (PSNR) can be mentioned [2]. These methods give equal importance to all pixels in the image during the computation of quality assessment metrics. Hence, they are generally not correlated with subjective image quality assessment methods [25]. They are used because they are based on the energy of the error signal (difference in signal between the images being compared), which is preserved after linear unitary (orthogonal) transforms such as Discrete Cosine Transform (DCT). The MSE and PSNR are computed using the following equations [9].

$$
\begin{gathered}
M S E=\frac{\sum_{i=1, j=1, k=1}^{M, N, 3}\left(X_{i, j, k}-Y_{i, j, k}\right)^{2}}{M \times N \times 3} \\
P S N R=10 \log _{10}\left(\frac{255^{2}}{M S E}\right)
\end{gathered}
$$

Where $X_{i, j, k}$ is the pixel value of the reference image, $Y_{i, j, k}$ is the pixel value of the decompressed image, $M, N$ are sizes of the image, $i, j$ are spatial coordinates of the image array and $k$ is the index of the color components of a color image.

As described above, the pixel value error in the corner regions of an endoscopic image during the compression process is zero, but the number of pixels shown in equation (2) includes the corner pixels. This reduces the MSE and increases the PSNR which makes these methods less correlated with the subjective image quality assessment method.
To alleviate this drawback, the modified versions of mean squared error ( $M S E \_W C E$ ) and peak signal-to-noise ratio $\left(P S N R_{-} W C E\right)$ are given below.

$$
M S E \_W C E=\frac{\sum_{i=0, j=0, k=0}^{M, N, 2}\left(X_{i, j, k}-Y_{i, j, k}\right)^{2}}{M \times N \times 3-3 \times N_{c}}
$$

Where $N_{c}$ represents the number of corner pixels.

$$
P S N R \_W C E=10 \log _{10}\left(\frac{255^{2}}{M S E \_W C E}\right)
$$

The number of corner pixels, in equation (4), includes those pixels whose values are unaltered by the image decompression process. It should be noted that the corner pixel values which are near to the boundary between the circular visual region and corner regions are affected by the reconstruction process of the lossy image decompression algorithms.

Furthermore, the effect of the pixels in the corner regions on the objective image quality assessment methods can also be investigated using the structural similarity index (SSIM). It is given by the following equation [10].

$$
S(X, Y)=\frac{\left(2 \mu_{x} \mu_{y}+C_{1}\right)\left(2 \delta_{x y}+C_{2}\right)}{\left(\mu_{x}^{2}+\mu_{y}^{2}+C_{1}\right)\left(\delta_{x}^{2}+\delta_{y}^{2}+C_{2}\right)}
$$

Where $\mu_{x}, \mu_{y}$ are the mean values, $\delta_{x}, \delta_{y}$ are the unbiased standard deviations of the reference and distorted images respectively, and $C_{1}, C_{2}$ are constants. From equation (6), we can conclude that the presence of zero-value corner pixels reduces the mean values and increases the values of variances in both the reference and distorted images. Moreover, the corner regions have no structural units that can be affected by compression algorithms. Hence, the structures in the decompressed and original images are similar in the corner regions of endoscopic images. This tends to increase the SSIM value, which is misleading.

To eliminate the effect of the corner regions, the pixel values in the circular visual regions only are included in the computation of IQA metrics. We clip the corner regions and compute the metrics. Overall, the presence of corner regions in capsule endoscopic images increases both PSNR and SSIM values during the evaluation of the quality metrics of the image compression algorithms. Hence, the exclusion of the corner regions in the image quality assessment metrics computation of wireless capsule endoscopy images makes the metrics more reliable. This is due to the fact that non-informative regions are not affected by the image compression process, and it increases the image quality scores of the IQA methods.

\section{B. The Effect of Boundary Region}

Unlike natural image boundaries, the boundary between corner regions and the circular visual region has a sharp pixel value transition. Consequently, it contains large highfrequency components when a block-based transform image compression algorithm is applied. The high-frequency components are quantized using larger quantizer values in such algorithms. Hence, this region is highly distorted by image compression algorithms. The highest distortion occurs in this region in both DPCM-based image compression algorithms that employ sub-sampling [2] and transform coding (DCT and 
DWT) methods [15], [16]. This is due to the fact that there is little correlation between the corner pixel values and those in the visual region.

The drawback of the existing objective image quality assessment methods for WCE is that both the PSNR and the SSIM values decrease as a result of the artificial boundary. This leads to unrealistic quality metrics value. Fig. 3 shows the scaled structural similarity map of the image given in Fig. 2(a). As we can see from the figure, the boundary between the corner regions and the circular visual region shows a very high difference. Unlike the rest of the region, the boundary region pixels are uncorrelated. This leads to significantly large highfrequency components in block-based transforms. Similarly, the uncorrelated nature of the pixels leads to a large error in subsampling based image compression algorithms. However, the distortion in this region has no effect on the quality of the visual perception of the image for of gastrointestinal abnormalities.

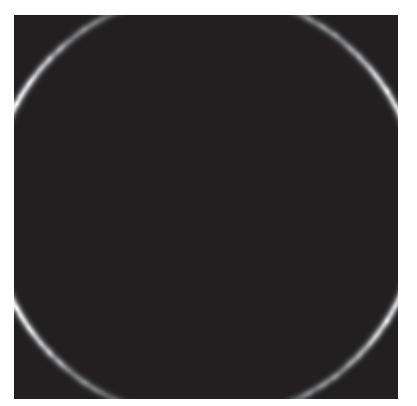

Fig. 3. The structural similarity map of the image shown in Fig. 2 (a).

Similar to the corner regions, the proposed solution in this work is the exclusion of the boundary region in the computation of IQA metrics. In this particular case, threadlike region whose width is 17 pixels ( 8 pixels into the corner region and 8 pixels into the visual region) perpendicular to the boundary is considered as a boundary region. The exclusion of the boundary region pixels helps us achieve reasonable image quality assessment metrics for WCE.

\section{Results AND Discussions}

The experimental setup, the results obtained from the experiments, and the analysis of the results are discussed in this section.

\section{A. Experimental Setup}

Extensive experiments were performed to compare the proposed IQA methods with general-purpose IQA methods. The experiments were designed and implemented by modifying the existing lossy image compression algorithms, which were proposed for WCE application. These image compression algorithms use DCT [15], DWT [16], and DPCM with subsampling [2] based image processing methods. The comparative analysis of the general-purpose and the proposed IQA methods was performed. The two selected IQA methods used in this work for demonstration purposes are PSNR and SSIM These metrics are chosen here because they are widely used in the performance analysis of image compression algorithms for WCE. In addition to that, the two methods are good representatives of the two categories of objective IQA algorithms. PSNR is based on the statistical error, and SSIM is based on the HVS perception [26], [27]. The main goal of these experiments is to prove the concept in practical scenarios using comparative analysis. It is not to compare the performance of different image compression algorithms.

In order to evaluate the performance of the proposed IQA methods, 120 images from Gastrolab [24] were selected. The selected images are in RGB format with different resolutions. The images from this database were used in the performance evaluation of several image compression algorithms [2], [17].

\section{B. Analysis of Results}

The comparison of mean squared error (MSE) of the pixel values in the boundary region and those inside the visual area are shown in Fig. 4. As shown in the figure, the average MSE values of pixels in the boundary region are 54.82, and those of the pixel values inside the visual area are 3.55 for standard JPEG image compression algorithm with quantizer value of 1.5. This shows that the pixels around the boundary region are

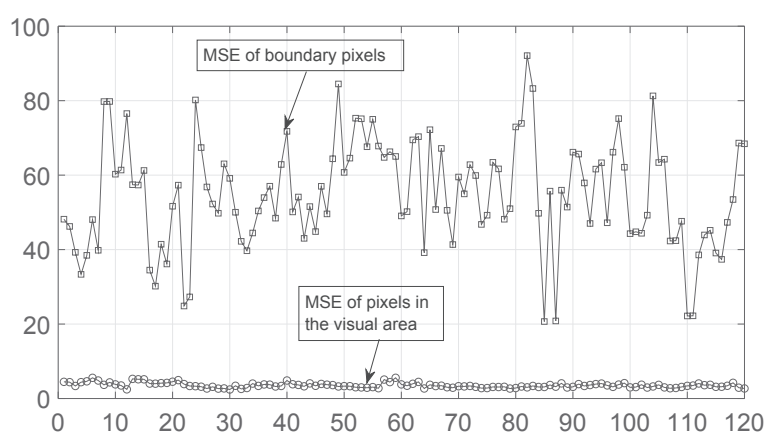

Fig. 4. The mean square error value of the pixels in the boundary and those in the visual region of 120 endoscopic images for standard JPEG image compression algorithm with a quantizer value of 1.5 .

highly affected by the compression algorithms due to the lack of correlation of the pixel values in the two regions. This leads to IQA metrics, which has less correlation with the subjective image quality assessment method.

The performance of the proposed ad-hoc IQA methods are evaluated using the implementation of eight different image compression algorithms which were proposed for WCE application. Both the general-purpose and the proposed IQA methods were implemented for comparative analysis. The summary of the comparison is given in Table I. As compared to the conventional IQA methods, the proposed algorithm that employs only the exclusion of corner regions in the IQA metrics computation achieves lower values of PSNR and SSIM. When the corner region pixels, which are not affected by the image compression process, are excluded from the computation of the IAQ metrics, the MSE increases as given in equations 2 and 4. This, in turn, leads to a lower PSNR value in all the image compression algorithms as shown in equations 3 and 5. On the other hand, the exclusion of 


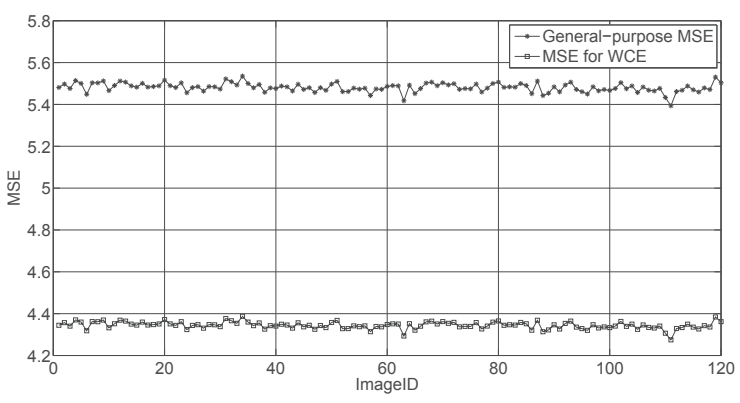

(a) Mean squared-error for 120 test images [24].

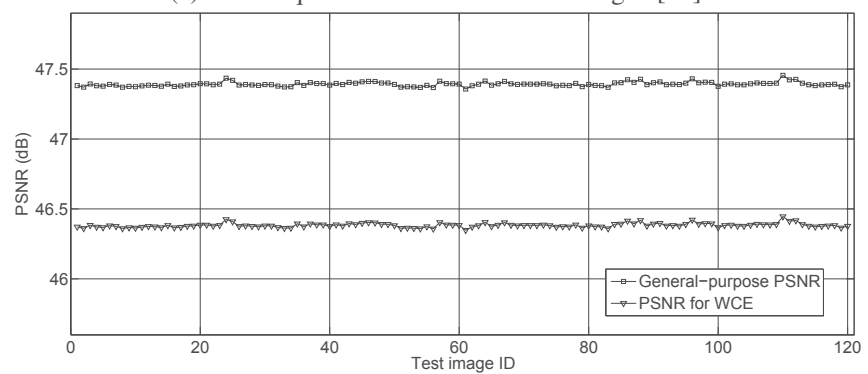

(b) PSNR value for 120 test images [24].

Fig. 5. Comparison of the MSE and PSNR values for the conventional and proposed IQA methods.

TABLE I

THE COMPARISON OF DIFFERENT IMAGE QUALITY ASSESSMENT METHODS.

\begin{tabular}{|c|c|c|c|c|c|c|}
\hline \multirow{3}{*}{$\begin{array}{l}\text { Image } \\
\text { Compression } \\
\text { Methods }\end{array}$} & \multicolumn{2}{|c|}{$\begin{array}{l}\text { Conventional } \\
\text { IQA Methods }\end{array}$} & \multicolumn{4}{|c|}{$\begin{array}{c}\text { Proposed } \\
\text { IQA Methods for WCE }\end{array}$} \\
\hline & \multirow[t]{2}{*}{$\begin{array}{l}\text { PSNR } \\
(\mathrm{dB})\end{array}$} & \multirow[t]{2}{*}{ SSIM } & \multicolumn{2}{|c|}{$\begin{array}{l}\text { Corner } \\
\text { Clipping }\end{array}$} & \multicolumn{2}{|c|}{$\begin{array}{l}\text { Corner Clipping } \\
\text { and Boundary } \\
\text { Region Exclusion }\end{array}$} \\
\hline & & & $\begin{array}{l}\text { PSNR } \\
(d B)\end{array}$ & SSIM & $\begin{array}{l}\text { PSNR } \\
(\mathrm{dB})\end{array}$ & SSIM \\
\hline $\begin{array}{l}\text { JPEG-based } \\
{[3]}\end{array}$ & 30.79 & 0.9998 & 30.07 & 0.9997 & 36.38 & 0.9997 \\
\hline $\begin{array}{l}\text { DPCM-based } \\
\text { [2] }\end{array}$ & 38.23 & 0.9996 & 37.50 & 0.9995 & 41.65 & 0.9998 \\
\hline $\begin{array}{l}\text { DCT-based } \\
{[15]}\end{array}$ & 38.62 & 0.9987 & 37.89 & 0.9985 & 38.94 & 0.9984 \\
\hline $\begin{array}{l}\text { DWT-based } \\
{[16]}\end{array}$ & 39.90 & 0.9899 & 39.42 & 0.9894 & 40.56 & 0.9905 \\
\hline $\begin{array}{l}\text { Modified } \\
\text { H.264 [28] }\end{array}$ & 38.29 & 0.9990 & 37.57 & 0.9988 & 37.76 & 0.9988 \\
\hline $\begin{array}{l}\text { DCT-based } \\
\text { [29] }\end{array}$ & 43.60 & 0.9995 & 42.88 & 0.9994 & 43.66 & 0.9994 \\
\hline $\begin{array}{l}\text { DCT-based } \\
{[30]}\end{array}$ & 31.45 & 0.9991 & 30.73 & 0.9991 & 31.38 & 0.9995 \\
\hline $\begin{array}{l}\text { DCT and } \\
\text { sub-sampling } \\
{[23]}\end{array}$ & 35.99 & 0.9049 & 35.26 & 0.9009 & 36.65 & 0.9061 \\
\hline Average & 37.11 & 0.9863 & 36.42 & 0.9857 & 38.37 & 0.9865 \\
\hline
\end{tabular}

the boundary region which is highly affected by the image compression processes reduces the MSE value and increases the PSNR value. In a nutshell, the exclusion of the corner regions alone reduces the PSNR value, whereas the removal of corner pixels alone increases the PSNR value. For the eight image compression algorithms implemented shown in Table I, the exclusion of corner regions in the computation of PSNR alone reduces it from $37.11 \mathrm{~dB}$ to $36.42 \mathrm{~dB}$ on average. Similarly, the exclusion of both corner regions and boundary region increases the PSNR value to $38.37 \mathrm{~dB}$ on average. This gives an improvement of $1.12 \mathrm{~dB}$ average value of PSNR. Hence, the combined effect is dominated by the impact of the boundary region between the corner regions and the visual region because this region is highly distorted by most the image compression algorithms due to the existence of sharp transition of pixel values at the boundary which leads to large values of high frequency components. This is consistent in all the images, compression algorithms. The observed PSNR value difference obtained differs from one algorithm to another since different algorithms induce a different level of distortion to the boundary region of WCE image.

Unlike the PSNR, SSIM value depends on image contrast, hue, and distribution of error [26], [27]. Because of this, we have not obtained a consistent pattern of changes in all image compression algorithms shown in Table I. On average, it is observed that the SSIM decreases from 0.9863 to 0.9857 with application of only corner clipping algorithm. It has increased to 0.9865 of average SSIM value with both corner clipping and boundary region omission in the computation of objective IQA for WCE images.

Obviously, the level of distortion of the corners pixels is zero since all the pixel values are zero. As a result, the average SSIM and PSNR values reduce when the corner region pixels are omitted in the computation of IQA metrics. The overall effect of corner clipping is decreasing the considered IQA metrics on average. The IQA metrics, which exclude only the boundary regions in the IQA computations, achieve a lower value of MSE compared to the original value as depicted in Fig. 4. Hence, it results in higher values of PSNR and SSIM than those of the conventional methods. This is due to the fact that the boundary region is highly affected by the image compression processes. The quantization and sub-sampling algorithms introduce high error at the boundary between the informative and non-informative regions due to the abrupt transition of intensity values of pixels in WCE images, as shown in Fig. 3. The distortion in these regions has no significance for the detection of abnormalities in this particular application. The modified IQA metrics, which are implemented with a combination of both corner clipping and boundary region exclusion in IQA metrics computation, show more realistic metric values than the conventional methods. Generally, the cumulative effect of excluding both regions is dominated by the impact of the boundary pixels. This shows that the distortion that occurs in the boundary region dominates the overall process, and the proposed IQA metrics are higher than that of the general-purpose IQA metrics. The simulation result shows consistent results for the majority of the evaluated image compression algorithms and test images, as shown in Fig. 5 (b).

Overall, more consistent image quality assessment metrics that are more correlated with subjective image quality assessment are proposed in this work, which can be reliably used in the image compression and super-resolution algorithm optimization process. The exclusion of the informative regions in the computation of the image quality assessment methods can sightly increase the computational complexity of the objective image quality assessment methods. However, with 
the workstation computational power, we have now, its impact in practical scenarios is negligible in terms of delay.

\section{Conclusion}

In this paper, an application-specific objective image quality assessment method for wireless capsule endoscopy is presented. The proposed method uses knowledge about the statistics of the image acquisition system to improve the performance of the general-purpose image quality assessment methods. Specifically, the corner regions, which are not affected by image compression algorithms, and the boundary regions between informative and non-informative regions, which are highly distorted by the image compression algorithms, are excluded in the computation of the objective image quality assessment metrics. The distortion in these two regions has no significance for the detection of abnormalities in this particular application. Hence, the proposed algorithm has a higher correlation to subjective quality assessment than the general-purpose IQA metrics. The overall impact of excluding the two regions in the computation of IQA metrics is that the general-purpose image quality measures are lower than the IQA measures proposed for WCE particular application. The proposed IQA method can be used in the optimization of image compression and image super-resolution algorithms. The proposed algorithm can easily be applied to other image quality assessment methods such as FSIM [11] etc.

\section{REFERENCES}

[1] G. Iddan, G. Meron, A. Glukhovsky, and P. Swain, "Wireless capsule endoscopy," Nature, vol. 405, no. 6785, pp. 417-417, 2000.

[2] K. A. Fante, B. Bhaumik, and S. Chatterjee, "Design and implementation of computationally efficient image compressor for wireless capsule endoscopy," Circuits, Systems, and Signal Processing, vol. 35, no. 5, pp. 1677-1703, 2016.

[3] C. Cheng, Z. Liu, C. Hu, and M. Q.-H. Meng, "A novel wireless capsule endoscope with jpeg compression engine," in 2010 IEEE International Conference on Automation and Logistics. IEEE, 2010, pp. 553-558.

[4] Y. Wang, C. Cai, and Y. Zou, "Single image super-resolution via adaptive dictionary pair learning for wireless capsule endoscopy image," in 2015 IEEE International Conference on Digital Signal Processing (DSP). IEEE, 2015, pp. 595-599.

[5] P. A. Floor, I. Farup, M. Pedersen, and $\varnothing$. Hovde, "Error reduction through post processing for wireless capsule endoscope video," EURASIP Journal on Image and Video Processing, vol. 2020, pp. 1-15, 2020.

[6] S. Charfi and M. El Ansari, "Computer-aided diagnosis system for colon abnormalities detection in wireless capsule endoscopy images," Multimedia Tools and Applications, vol. 77, no. 3, pp. 4047-4064, 2018.

[7] R. Istepanian, N. Philip, M. Martini, N. Amso, and P. Shorvon, "Subjective and objective quality assessment in wireless teleultrasonography imaging," in 2008 30th Annual International Conference of the IEEE Engineering in Medicine and Biology Society. IEEE, 2008, pp. 53465349.

[8] Z. Wang and A. C. Bovik, "Modern image quality assessment," Synthesis Lectures on Image, Video, and Multimedia Processing, vol. 2, no. 1, pp. 1-156, 2006.

[9] J. Korhonen and J. You, "Peak signal-to-noise ratio revisited: Is simple beautiful?" in 2012 Fourth International Workshop on Quality of Multimedia Experience. IEEE, 2012, pp. 37-38.

[10] Z. Wang, A. C. Bovik, H. R. Sheikh, and E. P. Simoncelli, "Image quality assessment: from error visibility to structural similarity," IEEE transactions on image processing, vol. 13, no. 4, pp. 600-612, 2004.

[11] L. Zhang, L. Zhang, X. Mou, and D. Zhang, "Fsim: A feature similarity index for image quality assessment," IEEE transactions on Image Processing, vol. 20, no. 8, pp. 2378-2386, 2011.
[12] M.-C. Lin, L.-R. Dung, and P.-K. Weng, "An ultra-low-power image compressor for capsule endoscope," BioMedical Engineering OnLine, vol. 5, no. 1, pp. 1-8, 2006.

[13] P. C. Cosman, R. M. Gray, and R. A. Olshen, "Evaluating quality of compressed medical images: Snr, subjective rating, and diagnostic accuracy," Proceedings of the IEEE, vol. 82, no. 6, pp. 919-932, 1994.

[14] L. He, F. Gao, W. Hou, and L. Hao, "Objective image quality assessment: a survey," International Journal of Computer Mathematics, vol. 91, no. 11, pp. 2374-2388, 2014.

[15] P. Turcza and M. Duplaga, "Low power fpga-based image processing core for wireless capsule endoscopy," Sensors and Actuators A: Physical, vol. 172, no. 2, pp. 552-560, 2011.

[16] K. A. Fante, B. Bhaumik, and S. Chatterjee, "A low-power color mosaic image compressor based on optimal combination of 1-d discrete wavelet packet transform and dpcm for wireless capsule endoscopy." in BIODEVICES, 2015, pp. 190-197.

[17] I. Intzes, H. Meng, and J. Cosmas, "An ingenious design of a high performance-low complexity image compressor for wireless capsule endoscopy," Sensors, vol. 20, no. 6, p. 1617, 2020.

[18] C. Babu, D. A. Chandy, and P. Karthigaikumar, "Novel chroma subsampling patterns for wireless capsule endoscopy compression," Neural Computing and Applications, vol. 32, no. 10, pp. 6353-6362, 2020.

[19] B. Sushma and P. Aparna, "Distributed video coding based on classification of frequency bands with block texture conditioned key frame encoder for wireless capsule endoscopy," Biomedical Signal Processing and Control, vol. 60, p. 101940, 2020.

[20] M. A. Usman, M. R. Usman, and S. Y. Shin, "Quality assessment for wireless capsule endoscopy videos compressed via hevc: From diagnostic quality to visual perception," Computers in biology and medicine, vol. 91, pp. 112-134, 2017.

[21] M. A. Usman and M. G. Martini, "On the suitability of vmaf for quality assessment of medical videos: Medical ultrasound \& wireless capsule endoscopy," Computers in biology and medicine, vol. 113, p. 103383, 2019.

[22] L. Lévêque, H. Liu, S. Baraković, J. B. Husić, M. Martini, M. Outtas, L. Zhang, A. Kumcu, L. Platisa, R. Rodrigues et al., "On the subjective assessment of the perceived quality of medical images and videos," in 2018 Tenth International Conference on Quality of Multimedia Experience (QoMEX). IEEE, 2018, pp. 1-6.

[23] M.-C. Lin and L.-R. Dung, "A subsample-based low-power image compressor for capsule gastrointestinal endoscopy," EURASIP Journal on Advances in Signal Processing, vol. 2011, no. 1, p. 257095, 2011.

[24] "Gastrolab," date last accessed January 10,2020. [Online]. Available: http://gastrolab.net/

[25] Q. Huynh-Thu and M. Ghanbari, "The accuracy of psnr in predicting video quality for different video scenes and frame rates," Telecommunication Systems, vol. 49, no. 1, pp. 35-48, 2012.

[26] A. Hore and D. Ziou, "Image quality metrics: Psnr vs. ssim," in 2010 20th international conference on pattern recognition. IEEE, 2010, pp. 2366-2369.

[27] Z. Kotevski and P. Mitrevski, "Experimental comparison of psnr and ssim metrics for video quality estimation," in International Conference on ICT Innovations. Springer, 2009, pp. 357-366.

[28] L.-R. Dung, Y.-Y. Wu, H.-C. Lai, and P.-K. Weng, "A modified h. 264 intra-frame video encoder for capsule endoscope," in 2008 IEEE Biomedical Circuits and Systems Conference. IEEE, 2008, pp. 61-64.

[29] P. Turcza and M. Duplaga, "Hardware-efficient low-power image processing system for wireless capsule endoscopy," IEEE journal of biomedical and health informatics, vol. 17, no. 6, pp. 1046-1056, 2013.

[30] K. Wahid, S.-B. Ko, and D. Teng, "Efficient hardware implementation of an image compressor for wireless capsule endoscopy applications," in 2008 IEEE International Joint Conference on Neural Networks (IEEE World Congress on Computational Intelligence). IEEE, 2008, pp. 27612765. 


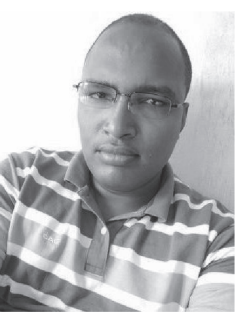

Kinde A. Fante received the BSc degree in Electrical Engineering from Bahir Dar University in 2008 the M.Tech degree in Electronics and Computer Engineering from Addis Ababa University, Ethiopia, in 2010,Ethiopia and the Ph.D. degree in Electrica Engineering from the Indian Institute of Technology Delhi, India, in 2016. Currently, he is an Assistant Professor in the Faculty of Electrical and Computer Engineering, Jimma Institute of Technology, Jimma University. His research interests include Biomedical Signal processing, low-power integrated circuits VLSI design for real-time image and video processing, and machine learning.

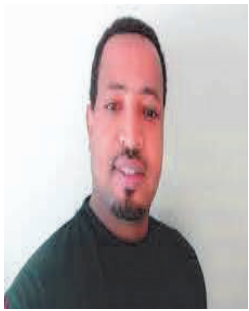

Fetulhak Abdurahman received the BSc in Electrical Engineering in 2009 and the MSc in Computer Engineering in 2014 from Addis Ababa University. From 2009- 2020 working as a lecturer in Jimma University, Ethiopia. His research interest includes computer vision, pattern recognition and natural language processing.

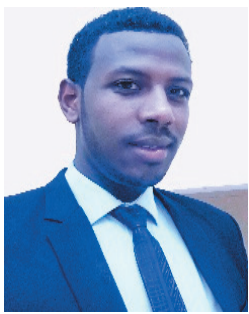

Mulugeta T. Gemeda received a BSc degree in Electrical and Computer Engineering in 2017 and M.Sc. degree in Electronics Communication Engineering from Jimma University, Ethiopia, in 2019. Currently, he is working as Lecturer at Jimma Institute of Technology in the Faculty of Electrical and Computer Engineering, Jimma University. His research interests include antenna design, mobile communication, and computer networking. 